\title{
TECHNIQUES FOR HIGH ACCURACY RELATIVE AND ABSOLUTE LOCALIZATION OF TERRASAR-X / TANDEM-X DATA
}

\author{
Ulrich Balss, Michael Eineder, Thomas Fritz, Helko Breit, and Christian Minet \\ German Aerospace Center (DLR), Remote Sensing Institute \\ Wessling, Germany
}

\begin{abstract}
The German SAR (synthetic aperture radar) satellites TSX1 and TDX-1 outperform former civilian space-borne radar sensors in terms of geometric accuracy. Recently, both satellites constitute the first bistatic SAR system in space: TanDEM-X. With the high geometric accuracy of both satellites, the geolocation of ground targets has to accurately consider signal propagation effects and geodynamic effects which formerly were negligible. The prediction of expected radar positions is additionally complicated by the bistatic acquisition geometry of TanDEM-X. Here, we present our approach to cope with these demanding requirements. With the application of these techniques, measurements of the geometric accuracy of TSX-1 and TDX-1 reveal that their pixel localization accuracy is significantly better than in previous studies.
\end{abstract}

Index Terms - synthetic aperture radar (SAR), bistatic SAR, pixel localization accuracy, geodesy

\section{INTRODUCTION}

The German SAR (synthetic aperture radar) satellite TerraSAR-X (TSX-1), launched in June 2007, outperforms former civilian space-borne radar sensors in terms of geometric accuracy. TSX-1 and its twin satellite TDX-1, launched in June 2010, constitute the first bistatic SAR system in space: TanDEM-X. Within the framework of the TanDEM-X mission, usually only one satellite (active satellite) transmits and both satellites (active and passive satellite) receive.

With the high geometric accuracy of TSX-1 / TDX-1, several signal propagation effects and geodynamic effects such as solid earth tides, which formerly were negligible, have to be considered in the geolocation of ground targets [1][2]. In case of the TanDEM-X mission, additionally the bistatic acquisition geometry of the passive imaging channel turns the prediction of the expected radar position of a given ground target into a demanding task. All the more, the high relative localization accuracy between active and passive imaging channel requires geolocation accuracy below cen- timeter level. For this purpose, we developed a numerical approach which copes with the requirements.

Recent measurements on the absolute pixel localization of TSX-1 and TDX-1 which carefully take into account all mentioned effects - even continental drift, give new insights into the outstanding geometric accuracy of both satellites and allow the isolation of very small, bandwidth dependent effects which were undiscovered so far. Measurements on the relative pixel localization of both imaging channels, active and passive channel, of the bistatic TanDEM-X platform even reveal accuracy values at sub-centimeter level.

\section{MEASUREMENT METHOD}

\subsection{General Considerations}

In order to verify the pixel localization accuracy of TSX-1 and TDX-1, the radar range and azimuth times of corner reflectors in focused SAR image are compared with their expected values obtained from precise GPS measurements [3] of their positions and estimated propagation delays. The conversion of the spatial GPS coordinates into expected radar time coordinates is based on zero Doppler equations [4] and orbit interpolation of the satellites' positions during datatake acquisition.

Radar systems indirectly measure geometric distances by means of the travel time of radar pulses from radar transmitter to ground and back to the radar receiver. Usually, the conversion from travel time to geometric distance uses the velocity of light in a vacuum. However, electrons in the ionosphere and water vapor in the atmosphere introduce additional time variant signal delays which have to be taken into account. At the level of the geometric accuracy of TerraSAR-X and TanDEM-X, geodynamic effects like earth tides and continental drift shift the true position of a ground target by several centimeters over the course of a day or years, respectively [1]. The aforementioned geometric shifts and propagation delays require access to external data sources which are not included in the delivered TerraSAR$\mathrm{X}$ data products.

The effect of the continental drift requires a careful choice of geodetic coordinate system. While the orbit trajec- 

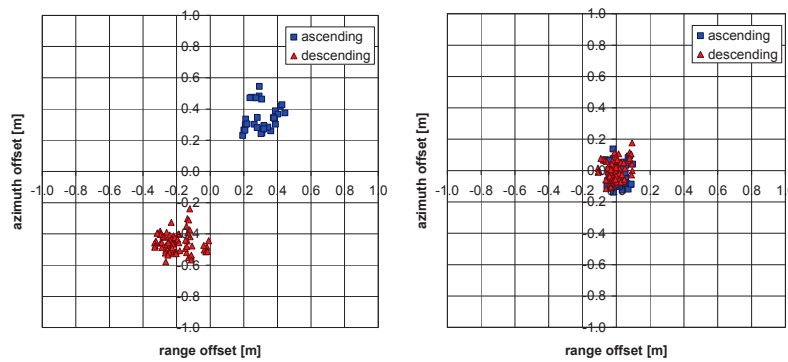

Figure 1: Effect of a coordinate system mismatch on the measured pixel localization error. Left: ETRS89 coordinates are misinterpreted as ITRS-2005 coordinates. Right: ETRS89 coordinates are correctly transformed to the ITRS2005 coordinate system.

tories of TSX-1 and TDX-1 are given in one of the international coordinate systems ITRS-2005 or ITRS-2008 ${ }^{1}$, for geodetic measurements a coordinate system which is fixed to the respective tectonic plate (e.g. ETRS89 in case of the Eurasian plate) is commonly used, even if these measurements are made with GPS. Therefore, in many cases a coordinate transform (e.g. available by [5]) is required before comparing ground and radar coordinates. If no coordinate transform is performed, a coordinate mismatch on the ground of approximately 0.6 meters between ETRS89 and ITRS-2005 is bound to happen and leads to a shift in the radar coordinates on the same order of magnitude.

Figure 1 illustrates the effect of such a coordinate mismatch on the measurement of pixel localization accuracy. The left plot of Figure 1 results if the ETRS89 coordinates of the corner reflectors are misinterpreted as ITRS-2005 coordinates. Because the Eurasian plate - and thus the ETRS89 coordinate system - drifts by about 3 centimeters per year to northeast [3], the expected corner positions shifted southwest. As a consequence, for ascending orbits where the satellite moves approximately from south to north, the sensor images a corner reflector somewhat later than expected. With right looking imaging geometry, there is also an apparent shift to far range. The opposite shifts in azimuth and range occur for descending orbits. Thus, an apparent incongruity in the measured corner positions between datatakes from ascending and descending orbits results. In contrast, the right plot in Figure 1 shows that with correctly transformed corner coordinates the measured corner positions in datatakes from ascending and descending orbits coincide.

\footnotetext{
${ }^{1}$ For datatakes which are recorded before April 17, 2011, the ITRS-2005 coordinate system is used. Later, the used coordinate system is ITRS-2008.
}
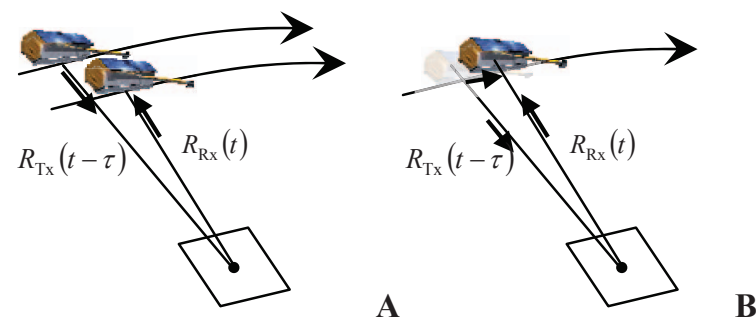

Figure 2: Bistatic acquisition geometry of TanDEM-X: passive (A) and active imaging channel (B).

\subsection{Specifics of Bistatic Acquisition Geometry}

In case of a bistatic acquisition geometry, the prediction of the expected slow and fast time position for a given ground target in a focused radar image has to consider the relative positions of three bodies which move relative to each other. There is the transmitting (active) satellite, the ground target and the receiving (passive) satellite. E.g. Figure 2A sketches the bistatic acquisition geometry of the passive imaging channel of TanDEM-X. Based on the sketch, the bistatic range history

$$
R(t)=\frac{1}{2} \cdot\left(R_{T x}(t-\tau(t))+R_{R x}(t)\right)
$$

of the given ground target results. It can be numerically computed by orbit interpolations for active and passive satellite. In order to cope with high accuracy requirements, equation (1) explicitly considers the signal travel time

(2) $\tau(t)=\frac{2 \cdot R(t)}{c}$

from the transmission of a radar pulse at the active satellite at slow time $t-\tau(t)$ to the reception of the radar echo at the passive satellite at slow time $t$. Because of the consideration of the signal travel time, equations (1) and (2) depend on each other, and the computation of the bistatic range history is in fact recursive. For this reason, we apply an iterative computation

$$
\begin{array}{ll}
\text { (3) } & R^{(0)}(t)=R_{R x}(t)+\Delta R_{\text {est }} \\
\text { (4 } & R^{(n+1)}(t)=\frac{1}{2} \cdot\left(R_{T x}\left(t-\frac{2 \cdot R^{(n)}(t)}{c}\right)+R_{R x}(t)\right) .
\end{array}
$$

The parenthesized upper index of $R^{(n)}(t)$ counts the iteration. $\Delta R_{\text {est }}$ is an initial estimate for the across-track baseline between both satellites. This term is not mandatory, but an adequate choice of $\Delta R_{\text {est }}$ improves the speed of convergence in the iterative computation. For moderate bistatic acquisition geometry like in the case of TanDEM-X, the shape of the range history resembles a hyperbola. The apex coordinates of the bistatic range history yield the slow $\left(t_{0}\right)$ and fast time $\left(\tau_{0}=2 \cdot R\left(t_{0}\right) / c\right)$ of bistatic closest approach. Because the range history is only numerically given, we numerically estimate the apex coordinates by nested intervals. 
It is not immediately obvious that these deductions refer also to the - apparently monostatic - one-satellite acquisition geometry of the active TanDEM-X channel. But, if there are sub-centimeter requirements on the pixel localization accuracy, even the motion of a single satellite between the instant of radar pulse transmission and the instant of echo reception gains relevance, and the stop-go approximation is no longer applicable. One has to explicitly distinguish between the satellite positions at both instants, as it is sketched in Fig. 2B. With this regard, even the one-satellite acquisition geometry has to be treated as bistatic geometry.

Due to these considerations, the true minimum signal travel time is slightly longer than expected from monostatic computation. It results when the satellite transmits a radar pulse approximately $\tau_{0} / 2$ before and receives the echo approximately $\tau_{0} / 2$ after the instant of minimum geometric distance between satellite and ground target. The exact proportion of transmit and receive path on the total signal travel time depends on the satellite's trajectory. In case of the acquisition geometry of the active TanDEM-X image channel, the range difference in the expected corner positions from both computation schemes, monostatic and bistatic, is typically in the order of magnitude of several tenth of a millimeter.

\section{ABSOLUTE LOCALIZATION ACCURACY}

We measured the absolute pixel localization accuracy of the TSX-1 satellite based on datatakes from the TerraSAR-X calibration campaigns 2007 and 2009. Figure 3 shows the measurement results. The range of the axes in the plots is chosen with regard to the TerraSAR-X product specification [6], which requires an accuracy $(1 \sigma)$ of 1 meter. Because of the accurate estimation of signal propagation effects by temporally and spatially adapted values and the consideration of geodynamic effects, our measurements reveal much better localization accuracy for TSX-1 than in previous studies (e.g. [7][8][9]).

With the increased measurement accuracy, small systematic effects previously hidden by the measurement error become visible. In particular, relative range offsets depending on the range bandwidth (100, 150 and $300 \mathrm{MHz}$, respectively) get apparent. The distribution of the localization error in the left plot of Figure 3 consists of three disjoint clusters which are shifted against each other in the range direction. Table 1 shows the average range offset of the centroid of each of these clusters. This bandwidth dependency can be currently corrected by the user and shall be taken into account in a future instrument and processor recalibration. With regard to the azimuth direction, the measured corner reflector positions are on average slightly shifted against the expected positions toward late azimuth by approximately 8 centimeters.
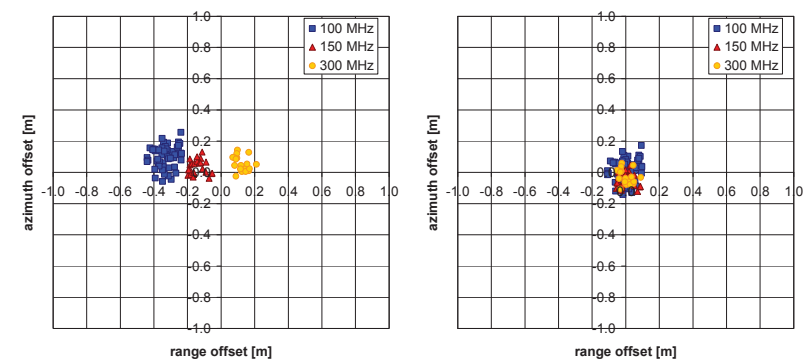

Figure 3: Pixel localization error of TSX-1. Left: based on current instrument calibration. Right: based on a modified instrument calibration which takes into account the bandwidth dependent range offset annotated in Table 1.

TABLE 1: BANDWIDTH DEPENDENCY OF THE MEASURED RANGE OFFSET FOR TSX-1 SATELLITE

\begin{tabular}{|l|l|}
\hline range bandwidth & remaining range offset \\
\hline $100 \mathrm{MHz}$ & $-33 \mathrm{~cm}$ \\
\hline $150 \mathrm{MHz}$ & $-14 \mathrm{~cm}$ \\
\hline $300 \mathrm{MHz}$ & $+12 \mathrm{~cm}$ \\
\hline
\end{tabular}

Until a product recalibration has been performed, the inverse of the range offsets in Table 1 yields an approximation for a range annotation shift which has to be applied in high accuracy measurements in order to compensate for this bandwidth dependency. The right plot of Figure 3 demonstrates the improved pixel localization accuracy which results with this correction and a correction of the measured average azimuth offset. Here, the previously disjoint clusters of measured values coincide with each other. The pixel localization error $(1 \sigma)$ improves to 5 centimeters in range and 7 centimeters in azimuth, respectively.

A large amount of this pixel localization error can be derived by theoretical considerations. According to [10], the expected accuracy of TerraSAR-X science orbits is about 4 centimeters. In the azimuth direction, the annotation accuracy of the datatake recording time provides an additional error contribution with 3.8 centimeters standard deviation. In azimuth, orbit and timing errors lead to an expected pixel localization error of approximately 5.5 centimeters. In range only the orbit error of 4 centimeters plays a role. From the results in [1] we expect that the remaining part of the pixel localization error can be predominantly attributed to the position determination error of the different corner reflectors.

\section{RELATIVE LOCALIZATION ACCURACY}

An important quality parameter for the co-annotation accuracy of active and passive imaging channel in bistatic TanDEM-X acquisitions is the relative offset of both channels. Table 2 shows first measurement results of this parameter based on bistatic acquisitions from the TanDEM-X commissioning phase. In azimuth, the position offset and its stan- 
TABLE 2: MEASURED PIXEL LOCALIZATION OFFSET OF TANDEM-X PASSIVE IMAGING CHANNEL RELATIVE TO THE ACTIVE CHANNEL IN SEVERAL BISTATIC ACQUISITIONS

\begin{tabular}{|l|l|l|l|l|}
\hline date & $\begin{array}{l}\text { active } \\
\text { satellite }\end{array}$ & $\begin{array}{l}\text { passive } \\
\text { satellite }\end{array}$ & $\begin{array}{l}\text { azimuth } \\
\text { offset } \\
{[\mathrm{mm}]}\end{array}$ & $\begin{array}{l}\text { Range } \\
\text { offset } \\
{[\mathrm{mm}]}\end{array}$ \\
\hline $\begin{array}{l}2010- \\
10-31\end{array}$ & TSX-1 & TDX-1 & $-25 \pm 16$ & $+10.3 \pm 1.1$ \\
\hline $\begin{array}{l}2010- \\
11-05\end{array}$ & TDX-1 & TSX-1 & $+141 \pm 36$ & $-3.5 \pm 0.9$ \\
\hline $\begin{array}{l}2010- \\
11-11\end{array}$ & TDX-1 & TSX-1 & $+16 \pm 18$ & $+4.6 \pm 1.5$ \\
\hline $\begin{array}{l}2010- \\
11-16\end{array}$ & TSX-1 & TDX-1 & $-36 \pm 30$ & $+0.4 \pm 1.5$ \\
\hline $\begin{array}{l}2010- \\
11-17\end{array}$ & TDX-1 & TSX-1 & $-10 \pm 62$ & $+0.6 \pm 0.4$ \\
\hline
\end{tabular}

dard deviation are in the order of magnitude of a few centimeters. However, the accuracy in range is significantly better. There are just a few millimeters of average range offset while the standard deviation is typically about 1 millimeter. While several sources of error (e. g. the signal propagation delays) are equal in both simultaneously acquired imaging channels, and thus their effect cancels out in a relative measurement, the outstanding performance is mainly based on the precise knowledge of the satellites' baseline at submillimeter level.

\section{CONCLUSIONS}

With the high geometric accuracy of TSX-1 / TDX-1, the geolocation of ground targets has to accurately consider signal propagation effects and geodynamic effects which were formerly negligible. Our recent measurements take these effects into account and reveal significantly better absolute pixel localization accuracy for TSX-1 and TDX-1 than previous studies.

Once signal propagation and geodynamic effects are thoroughly considered, small systematic effects which were hidden by measurement error, become visible and can be compensated for in future. The consideration of all of these effects enables absolute pixel localization accuracy on the order of magnitude of just a few centimeters.

In bistatic TanDEM-X acquisitions, the relative pixel localization accuracy in range is even better because on the one hand most of the remaining error contributions are equal in both image channels and thus cancel out in a relative measurement, and on the other hand the baseline between both satellites is more precisely known than the satellites' absolute positions. In this way, we reveal relative localization accuracy at sub-centimeter level.

\section{ACKNOWLEDGMENTS}

We thank our colleagues from the TerraSAR-X/TanDEM-X IOCS (Instrument Operations and Calibration Segment) team who granted us access to their precise GPS measurement of corner reflector positions and the datatakes of the TerraSAR-X and TanDEM-X calibration campaigns. We thank Sebastian Tuttas for his experiments and research that helped us, reveal the effect of the continental drift.

\section{REFERENCES}

[1] M. Eineder, X. Cong, C. Minet, P. Steigenberger, T. Fritz, and W. Abdel Jaber, "Imaging Geodesy with TerraSAR-X," 2010 IEEE International Geoscience and Remote Sensing Symposium, July, 25-30, 2010, Honolulu, USA.

[2] M. Eineder, C. Minet, P. Steigenberger, X. Cong, and T. Fritz, "Imaging Geodesy - Towards Centimetre-Level Ranging Accuracy with TerraSAR-X," Transactions on Geoscience and Remote Sensing, 2010.

[3] Tuttas, S., Joint Gravimetric and Geometric Survey of Geophysical Signals - Feasibility Study for the TERENO Alpine and Prealpine Ammer Observatory, master thesis, Technical University Munich, 2010.

[4] Cumming, I. G., and F. H. Wong, Digital Processing of Synthetic Aperture Radar Data: Algorithms and Implementations, Artech House, Boston, MA, 2005, ch. 4.2-4.3, pp. 114-129.

[5] EPN Central Bureau - Royal Observatory of Belgium: http://www.epncb.oma.be

[6] Fritz, T., and M. Eineder, TerraSAR-X Ground Segment Basic Product Specification Document, TX-GS-DD-3302, version 1.5, 2008.

[7] M. Schwerdt, B. Bräutigam, M. Bachmann, B. Döring, D. Schrank, and J. Hueso Gonzalez, "Final TerraSAR-X Calibration Results Based on Novel Efficient Methods," Transactions on Geoscience and Remote Sensing, vol. 48, No. 2, pp. 677-689, 2010.

[8] A. Schubert, M. Jehle, D. Small, and E. Meier, "Geometric Validation of TerraSAR-X High-Resolution Products," Proc. 3rd TerraSAR-X Sci. Team Meeting, Oberpfaffenhofen, Germany, 2008.

[9] A. Schubert, M. Jehle, D. Small, and E. Meier, "Influence of Atmospheric Path Delay on the Absolute Geolocation Accuracy of TerraSAR-X High Resolution Products," Transactions on Geoscience and Remote Sensing, vol. 48, No. 2, pp. 751-758, 2010.

[10] Y. Yoon, M. Eineder, N. Yague-Martinez, and O. Montenbruck, "Precise Trajectory Estimation and Quality Assessment," Transactions on Geoscience and Remote Sensing, vol. 47, No. 6, pp. 1859-1868, 2009. 